\title{
Three-stage features of loess dynamic deformation and estimation method on the residual strain
}

\author{
Junjie Sun ${ }^{\text {i) }}$, Zhijian Wu ${ }^{\text {ii) }}$ and Kun Liu ${ }^{\text {iii) }}$ \\ i) Professor, Lanzhou Institute of Seismology, CEA, 450, Donggangxilu Ave., Lanzhou 730000, China. \\ ii) Professor, Lanzhou Institute of Seismology, CEA, 450, Donggangxilu Ave., Lanzhou 730000, China.
}

iii) Assistant Professor, Key Laboratory of Loess Earthquake Engineering, Gansu Province \& CEA, 450, Donggangxilu Ave., Lanzhou 730000, China.

\begin{abstract}
Here we hoped to understand loess dynamic deformation through analyzing the physical process and mechanical mechanism. Therefore, we introduced a concept of stress ratio and proposed its mathematical formula to disclose the three-stage feature of loess dynamic strain hiding inside a series of laboratory data. By applied Mohr-Coulomb failure criterion, the stress ratio could consider three aspects of influence factors, i.e. structure strength of soil, consolidation pressure in actual field and seismic loading onto the soil. To a certain extent, the stress ratio could reveal the relative tolerability of loess soil under seismic loadings. Based on three-stage features of loess dynamic deformation, we obtained an empirical relation between stress ratio and compression value of void ratio. Then combined with a theoretical relation of dynamic residual strain, initial void ration and compression value of void ratio, we provided a semi-empirical model to estimate the dynamic residual strain of unsaturated loess.
\end{abstract}

Keywords: unsaturated loess, dynamic residual strain, influence parameter, stress ratio, magnitude estimation

\section{INTRODUCTION}

Loess is a kind of uniform, cohesive and wind-blown sediment within Quaternary period (Terzaghi et al., 1996). True loess deposits have never been saturated and its cohesion is due to the presence of a binder that may be predominantly calcareous or clayey. In case of saturation the bond between particles is weakened and the depositing surface may settle. Meanwhile, natural loess could suffer another obvious settlement under seismic loadings, so called dynamic residual deformation or seismic subsidence. Previous research results show that dynamic deformation of unsaturated loess may have relations with its high porosity and weak cohesion.

During the early stage, collapsibility is the main concerned problem in geotechnical practices of loess (Gao, 1980; Lei, 1987; Miao et al., 1999; Rogers et al., 1994). After the concept of seismic subsidence of loess was proposed in 1980s, dynamic residual deformation attracted more attention (Wang et al., 1993). At present loess dynamics has become a thriving topic in the field of geotechnical earthquake engineering. On the view of development level, loess collapsibility has been known well (Chen et al., 2013; Chu et al., 2014; Shao et al., 2014), but the understanding on dynamic residual deformation is still deficient (Sun et al., 2012). Because of the complexity of dynamic issues, some essential problems of loess seismic subsidence (e.g. critical influence parameters) are even indistinct, needless to say its plastic constitutive relation (Sun et al., 2012).

Natural loess especially of which deposited during $\mathrm{Q}_{3}$ period could suffer an obvious residual deformation due to gradually weaker structure strength caused by dynamic loadings. This means there is an initial dynamic stress to cause seismic subsidence. During the early research period, influence parameters are concerned. These works focus on the strength change associated with single factor, e.g. water content, void ratio, density, modulus of elasticity, and consolidation pressure. Of course, the seismic loading feature is another focal point, e.g. vibration times, loading types, predominant period, effective duration and peak value. For loess seismic subsidence, properties of soil mass and features of seismic loading are coequally important. However, the previous efforts are not enough on the analysis of physical process and mechanical mechanism (Deng et al., 2007; Wang et al., 1993; Wang et al., 2007). These results are unreasonable to acquire the knowledge of critical influence parameters because concerned factors are dependent each other, for example, the structure strength having relation with factors of water content, density, modulus of elasticity and consolidation pressure. Therefore, the work could not distinctly reveal the physical relationship between dynamic residual deformation and critical influence properties, especially the structure strength and 
under-compacted status, (Sun et al., 2013).

We hope to establish two aspects of relations of three-phase (air, water and solid) volume deformation and dynamic void compression, here, combining to describe dynamic behaviors of unsaturated loess. The first one reveals a theoretical relationship of dynamic residual strain, initial void ratio and compression value of void ratio. The second one shows an empirical relationship of compression value of void ratio, shear strength, consolidation pressure and seismic loading based on loess dynamic deformation data in laboratory. This work would be helpful to understand the reasonable and practical constitutive model of natural loess under external loadings.

\section{METHODOLOGY AND TESTING DATA}

\subsection{Laboratory data of unsaturated loess}

By means of laboratory tests, we acquired relative data of untrimmed samples, which were collected in a typical loess field (Lintao County of Gansu Province, China). Cohesion and internal friction angle were obtained by static triaxial apparatus, whereas loess residual strain by dynamic triaxial tests (see Fig. 1). Other data of physical properties including void ratio were expediently achieved through geotechnical tests in laboratory.

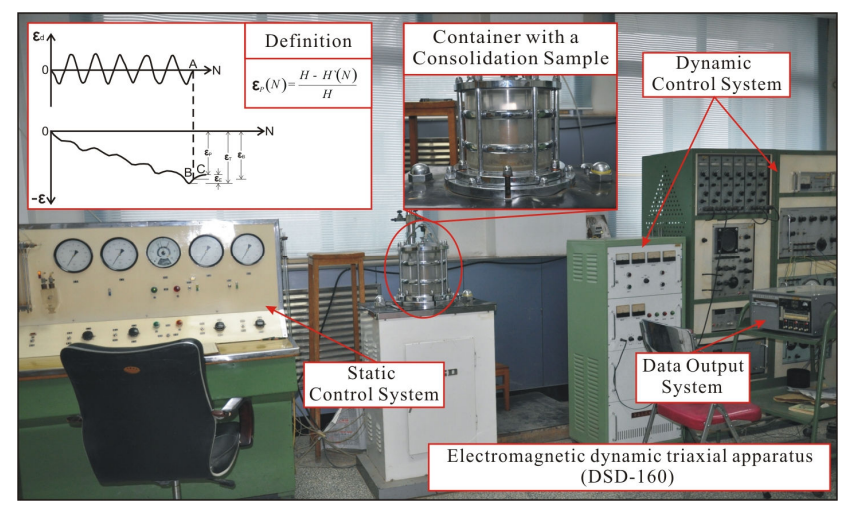

Fig. 1. Laboratory panoramic view of dynamic triaxial test and definition of dynamic residual strain of loess.

For dynamic triaxial tests (detailed method and procedure referring to Wang et al., 2003), confining pressures are jointly calculated by burial depth, unit weight and lateral-compression coefficient of loess samples. In this case, loess samples are confined because of actual stress conditions in field. Dynamic stress of each sample is experientially estimated due to confining pressures; and the stress axially exerts 60 times. We record one data of dynamic residual strain each 10 times; and finally there are 6 data for each untrimmed sample to describe its dynamic behaviour of residual deformation. Compression values of void ratio, meanwhile, are obtained by porous difference between before and after loaded.

Based on above-mentioned procedure of laboratory tests, the relative data of loess samples are summarized in Fig. 2 and Fig. 3.

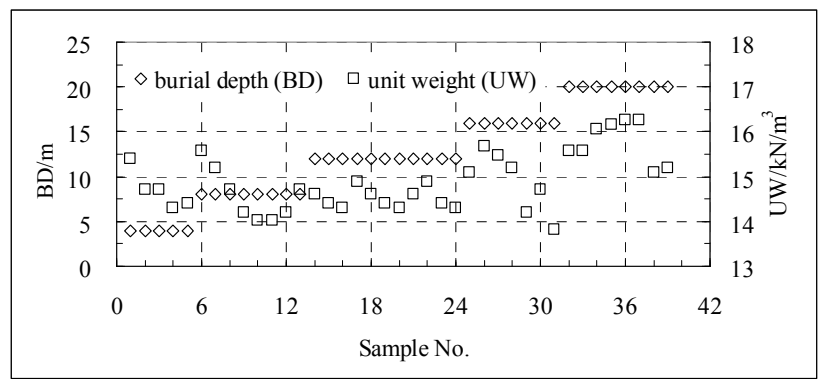

(a)

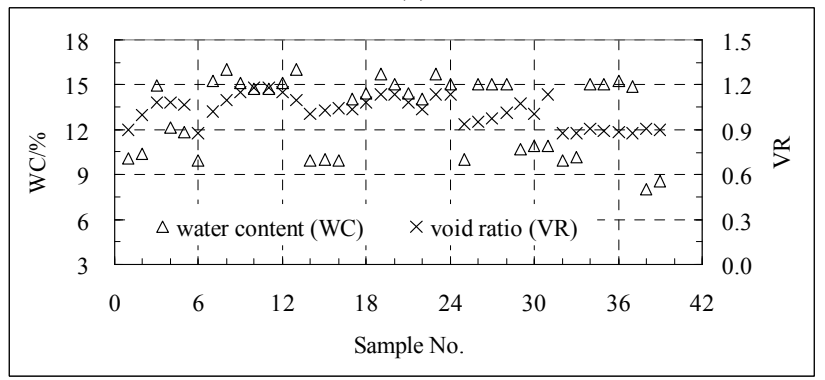

(b)

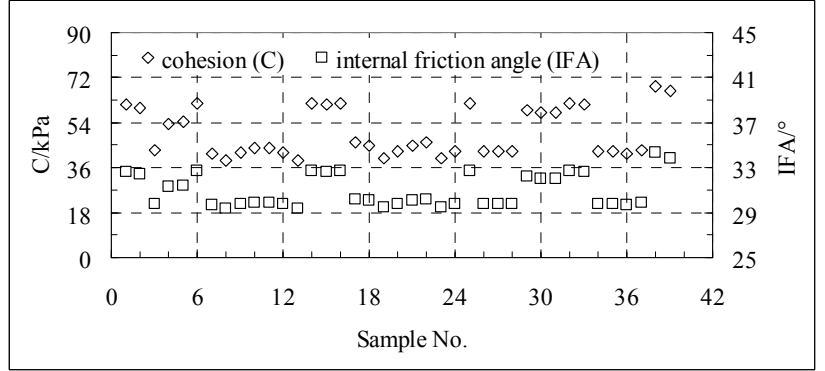

(c)

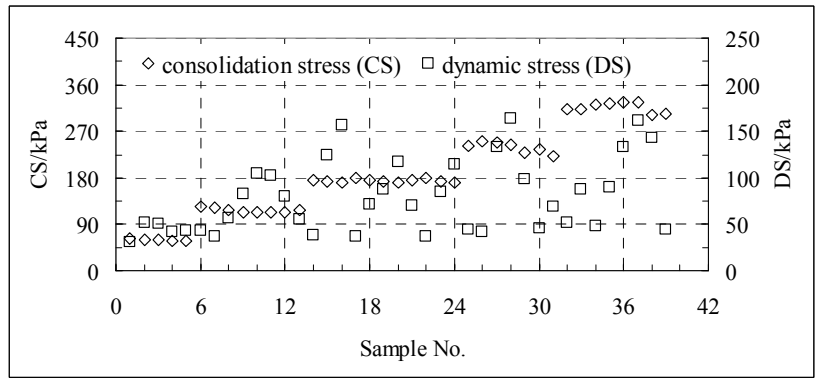

(d)

Fig. 2. Physical properties and loading stresses of loess samples.

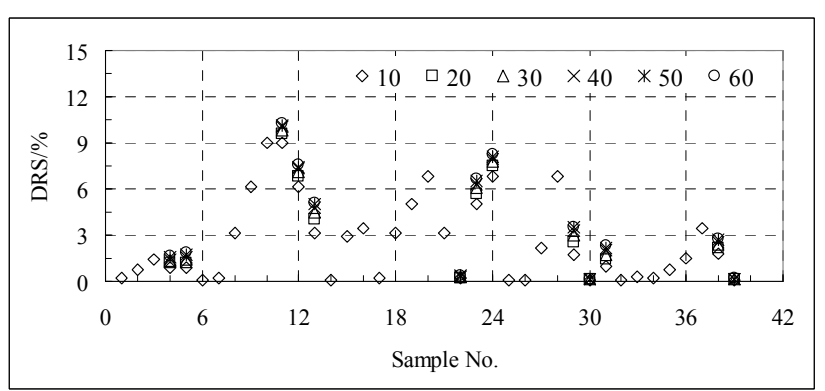

(a) dynamic residual strain (DRS) 


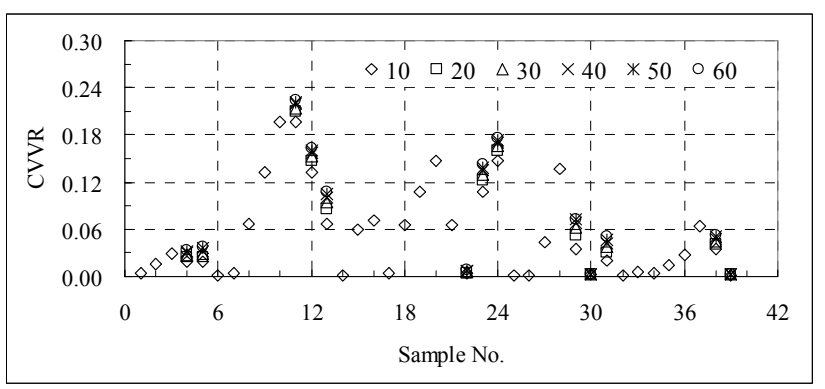

(b) compression values of void ratio (CVVR)

Fig. 3. Laboratory data of DRS (a) and CVVR (b) of loess samples under different loading times (e.g. 10, 20...60)

\subsection{Theoretical relationship of three-phase volume deformation of unsaturated loess}

Based on a procedure of theoretical analysis, here, the author hopes to provide a relationship between dynamic residual strain $(\varepsilon)$ and initial void ratio $\left(e_{i}\right)$ of unsaturated loess.

As shown in Fig. 4, we adopted an ideal model with the same area $(A)$ at the top and bottom, the height $(H)$, and the initial void ratio $\left(e_{i}\right)$ to reveal the relation of dynamic residual strain, initial void ratio and compression value of void ratio. After a seismic loading $(g(t))$ exerted, the analysis model would have a new area $\left(A^{\prime}\right)$ and height $\left(H^{\prime}\right)$, and a final void ratio $\left(e_{f}\right)$. For the model, volumes of single phase of air, water and solid and total void are respectively marked with $V_{a}, V_{w}, V_{s}$ and $V_{v}$.

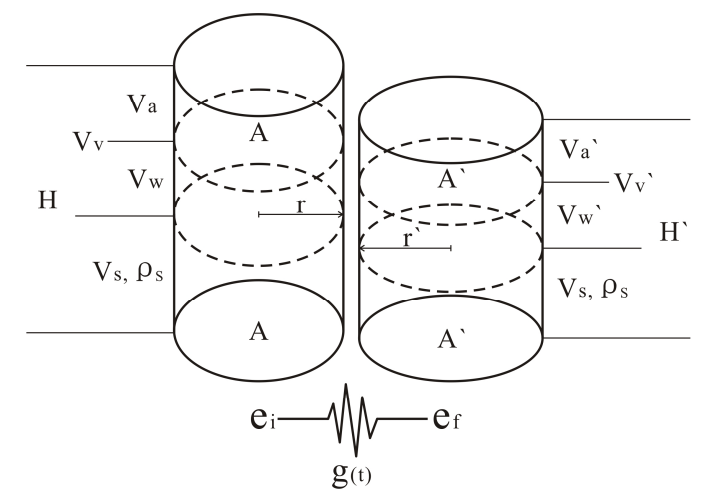

Fig. 4. Ideal model for volume deformation analysis of three phases of air, water and solid of unsaturated loess.

As the ideal model suffers residual strain under seismic loadings, compressive and tensile strains could respectively occur in axial and lateral directions. According to the definition of Poisson's ratio $(v)$, we obtain the equation

$$
v=\frac{\left(r^{\prime}-r\right) / r}{\left(H-H^{\prime}\right) / H}
$$

Based on the definition of dynamic residual strain (see Fig. 1), we achieve the equation

$$
\varepsilon=\left(H-H^{\urcorner}\right) / H
$$

From Eqn. (2), Eqn. (1) can be written as

$$
r^{\prime}=(1+v \varepsilon) r
$$

Considering three phases of air, water and solid, another expression of Eqn. (2) is

$$
\varepsilon=\frac{\left[\left(H_{a}+H_{w}\right)+H_{s}\right]-\left[\left(H_{a}{ }^{\prime}+H_{w}{ }^{\prime}\right)+H_{s}{ }^{\prime}\right]}{\left(H_{a}+H_{w}\right)+H_{s}}
$$

where $H_{a}, H_{w}$ and $H_{s}$ are respectively the heights of three phases of air, water and solid; and $V=H A=\left(H_{a}+H_{w}+H_{s}\right) A$.

From Eqn. (3), Eqn. (4) can be written as

$$
\varepsilon=\frac{\left[\left(V_{a}+V_{w}\right)+V_{s}\right]-(1+v \varepsilon)^{-2}\left[\left(V_{a}{ }^{\prime}+V_{w}{ }^{\prime}\right)+V_{s}{ }^{\prime}\right]}{\left(V_{a}+V_{w}\right)+V_{s}}
$$

Here, we suppose the solid volume does not alter in any case during dynamic residual deformation, a simple expression of Eqn. (5) is

$$
\varepsilon=1-\frac{(1+v \varepsilon)^{-2}\left[\left(V_{a}{ }^{\prime}+V_{w}{ }^{\prime}\right)+V_{s}{ }^{\top}\right]}{\left(V_{a}+V_{w}\right)+V_{s}}
$$

The definition of void ratio before and after loading exerts is respectively $e_{i}=V_{v} / V_{s}$ and $e_{i}=V_{v}{ }^{\prime} / V_{s}$. Thus a new expression of Eqn. (6) is

$$
\varepsilon=1-\frac{(1+v \mathcal{E})^{-2}\left[\left(e_{a f}+e_{w f}\right)+1\right]}{\left(e_{a i}+e_{w i}\right)+1}
$$

where $e_{a}$ and $e_{w}$ are respectively void ratios of two phases of air and water; and total void ratio of soil mass is $e=e_{a}+e_{w}$.

We suppose dynamic residual strain of unsaturated loess is only associated with the compression of void space and introduce a definition of compression value of void ratio $\left(\Delta e=e_{i}-e_{f}\right.$; here ignored the individual void compression of air and water). Eqn. (7) can be written as

$$
\varepsilon=1-(1+v \varepsilon)^{-2}\left(1-\Delta e /\left(e_{i}+1\right)\right)
$$

The simple expression of Eqn. (8) is

$$
v^{2} \varepsilon^{3}+\left(2 v-v^{2}\right) \varepsilon^{2}+(1-2 v) \varepsilon=\frac{\Delta e}{e_{i}+1}
$$

At the confined condition $(v=0)$, Eqn. (9) has a simplified expression as the follow.

$$
\varepsilon=\frac{\Delta e}{e_{i}+1}
$$

In this paper, the author adopts Eqn. (10) to describe dynamic residual deformation of unsaturated loess because the actual stress condition in soil field is approximately confined.

\subsection{Analysis method on empirical relationship of dynamic compression of loess's void}

Because of the complexity of dynamic residual 
deformation of unsaturated loess (see Fig. 5), the author analyzes the relationship of compression value of void ratio, structure strength, consolidation pressure and seismic loading based on laboratory data of loess samples. During analysis process, we introduce a concept of stress ratio $\left(R_{s}\right)$ to reflect combined influences of critical factors, i.e. structure strength of loess soil, consolidation pressure in actual field and seismic loading onto the soil.

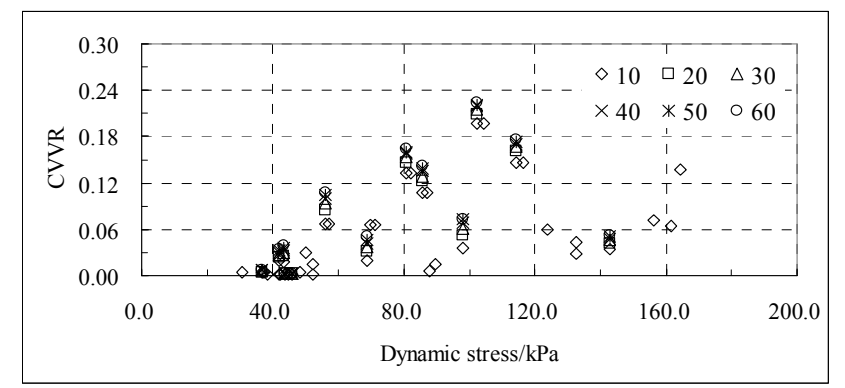

Fig. 5. Indistinct relation between dynamic stress and compression value of void ratio (CVVR).

The definition of $R_{s}$ is

$$
R_{s}=R\left(f\left(\sigma_{d}\right) / \tau_{c}\right)=f\left(\sigma_{d}\right) / \tau_{c}
$$

Considering consolidation stress $\left(\sigma_{c}\right)$ in actual field, the Mohr-Coulomb criterion can be written as

$$
\tau_{c}=C+\sigma_{c} \tan \varphi
$$

For single loess sample, compression value of void ratio gradually increases with loading times of dynamic stress and the development feature can be well described by the logarithmic function (see Fig. 6).

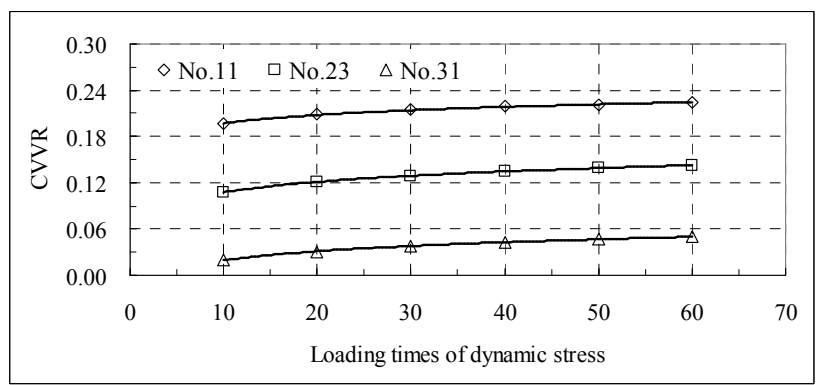

(a)

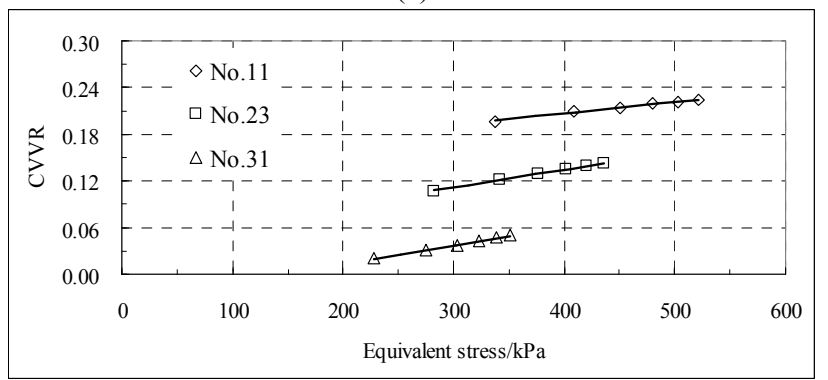

(b)

Fig. 6. Examples of relations between compression value of void ratio (CVVR) and loading times of dynamic stress (a) or equivalent stress (b) for single loess sample.
Consequently, we defined an equivalent stress $\left(f\left(\sigma_{d}\right)\right)$ to figure the influence of loading times of dynamic stress $(N)$ on a certain sample of unsaturated loess as the follow.

$$
f\left(\sigma_{d}\right)=(1+\ln (N)) \sigma_{d}
$$

From Eqn. (12) and Eqn. (13), the detailed expression of Eqn. (11) is

$$
R_{s}=\frac{[1+\ln (N)] \sigma_{d}}{C+\sigma_{c} \tan \varphi}
$$

\section{RESULTS}

\subsection{Checkout of laboratory data on the theoretical relation of Eqn. (10)}

As one of two essential relations to describe residual deformation of unsaturated loess under seismic loadings, the rationality of the theoretical relation of Eqn. (10) needs to be verified carefully.

Figure 7 shows the checkout results. The little difference between mean value and statistical slope reveals the rationality of Eqn. (10) is fine.

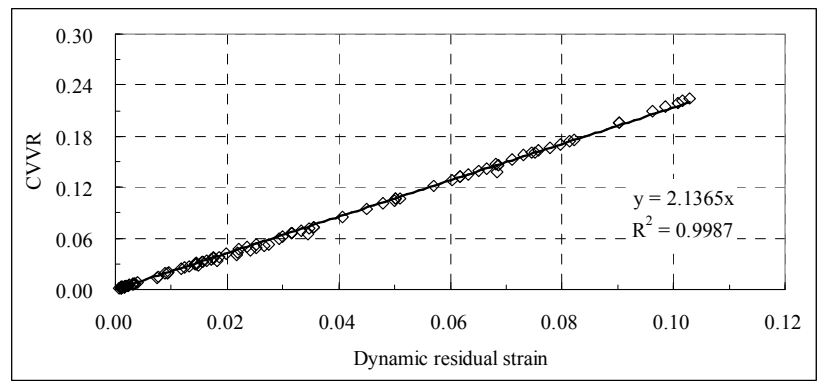

Fig. 7. Quantitative relationship of dynamic residual strain and compression value of void ratio (CVVR) for loess samples (Mean value of initial void ratio plus 1: 2.0239; statistical slope of testing data: 2.1365; the difference between mean value and statistical slope is around $5.6 \%$ ).

\subsection{Three-stage features of dynamic deformation of loess based on Eqn. (14)}

According to the relative laboratory data in Fig. 2 and Fig. 3, and the mathematical relation of Eqn. (14), we could obtain the key feature of residual deformation process of unsaturated loess under seismic loadings (see Fig. 8).

As shown in Fig. 8 (a), the deformation process obviously includes three stages (grey thick lines). STAGE I has a residual strain around 0 due to lower external loading than initial dynamic stress. Within STAGE III, the residual strain keeps a constant associated with ultimate feature of void compression. STAGE II is the most complex period of loess dynamic deformation. The data discreteness may be caused by calculation errors of Eqn. (14) due to laboratory data of cohesion and internal friction angle. Points of I-II and II-III figure the boundary between adjacent stages of dynamic void compression process. 


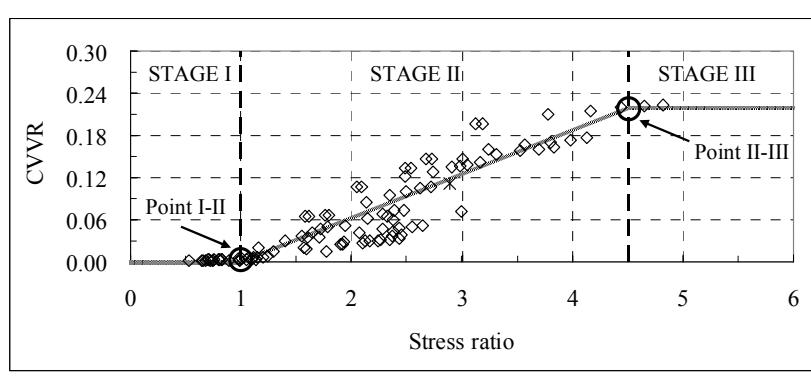

(a) three-stage features

(CVVR: compression value of void ratio)

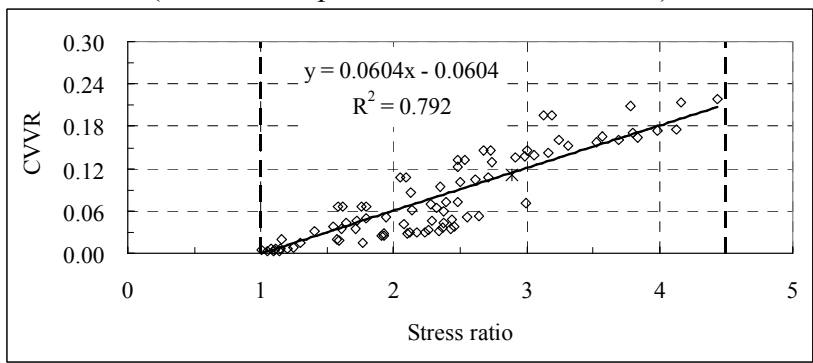

(b) STAGE II

(CVVR: compression value of void ratio)

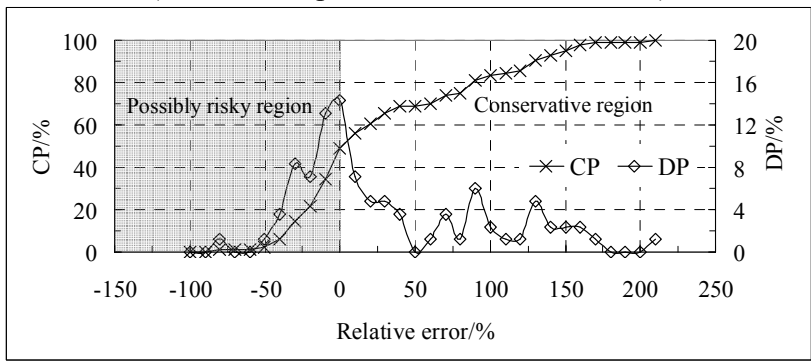

(c) STAGE II

(CP: cumulative probability; DP: distribution probability)

Fig. 8. Three-stage features of loess residual deformation under seismic loadings (a; the star expressing the centre of laboratory data within STAGE II), empirical relationship of the STAGE II (b), and its frequentness probability of relative error (c; calculation results of laboratory data within (b), detailed procedure referring to Eqn. (15) and Eqn. (16)).

Applied the statistical relationship in Fig. 8 (b), we can obtain a calculation result associated with each laboratory data of compression value of void ratio. The relative errors of calculation results are computed by the following equation.

$$
e_{r-i}=\frac{r_{i}-d_{i}}{d_{i}}
$$

where $e_{r-i}$ is the relative error; $r_{i}$ is the calculation result; and $d_{i}$ is the corresponding data in laboratory.

There are 84 data within STAGE II. We analyze the frequentness probability of these data according to the equation as follows.

$$
P_{\left(X_{k-1} \leq X<X_{k}\right)}=\frac{N_{\left(X_{k-1} \leq X<X_{k}\right)}}{N_{\text {total }}}
$$

where $X$ expresses the error values; $P$ is the probability value; $N$ is the number of error values within the interval of $X_{k-1}$ and $X_{k} ; N_{\text {total }}$ is total number of error values.

Within the possibly risky region (see Fig. 8(c)), the cumulative probability (CP) is around $48.8 \%$. This means the calculation result might be conservative or risky. Generally, the earthquake is a kind of small probability event. On the view of probability, we could choose linear relationship to describe complex STAGE II because conservative errors would be offset by possibly risky errors.

\subsection{Estimation method on dynamic residual strain of loess}

Based on Fig. 8, we can achieve an empirical relation between compression value of void ratio $(\Delta e)$ and stress ratio $\left(R_{s}\right)$ as the follow.

$$
\Delta e=0.06 R_{s}-0.06
$$

Generally, equivalent cyclic shear stress of seismic loadings $\left(\tau_{a v}\right)$ can be calculated by means of Seed's method (Seed et al., 1971) and the formula is

$$
\tau_{a v}=\frac{0.65 \gamma z r_{d} a_{\max }}{g}
$$

where $\gamma, z, r_{d}, a_{\max }$ and $g$ are respectively unit weight, burial depth, reduction coefficient of depth, horizontal component of peak ground acceleration and gravity acceleration.

During laboratory triaxial test, dynamic stress $\left(\sigma_{d}\right)$ is the double of equivalent cyclic shear stress $\left(\tau_{a v}\right)$ (Qian et al., 1996), which means

$$
\sigma_{d}=2 \tau_{a v}
$$

From Eqn. (10), Eqn. (14), and Eqn. (17)-(19), we could obtain an estimation method of dynamic residual strain of unsaturated loess with a semi-empirical formula as the follow.

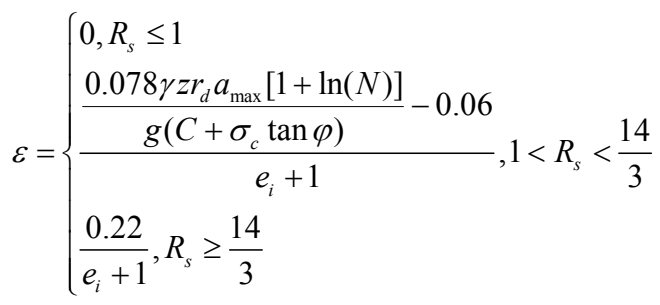

where the meaning of parameters is the same with preceding text.

According to laboratory data (see Fig. 8 (a)), the ultimate compression value of void ratio (CVVR) is about 0.22 (within STAGE III). The typical loess (with residual-strain ability under seismic loadings) generally has a value of void ratio around 1.0 and the loess could not suffer a dynamic residual strain while its void ratio less than 0.8 (Wang et al., 2003). Their difference between 1.0 and 0.8 is 0.2 (being close to above 0.22 ). To a certain extent, therefore, the value of 0.22 could reflect the maximum compression possibility of loess void. Supposing the ultimate CVVR is 0.22 in the case 
of loess dynamic deformation, we obtain the value of 14/3 in Eqn. (20) calculated by Eqn. 17.

\section{CONCLUSIONS}

Dynamic residual deformation of unsaturated loess is critically influenced by three aspects of factors, i.e., soil parameters, features of seismic loadings and stress state in soil field. Critical influence parameters of loess properties mainly include cohesion, internal friction angle and void ratio. Features of the seismic loading could be figured by peak value and duration of ground motion. The stress stated in soil field may be briefly described by consolidation stress.

The theoretical relation of dynamic residual strain and void ratio reveals the physical deformation process, which means the residual strain is distinctly caused by the compression magnitude of void space. Under a certain dynamic loading, the final compressive magnitude is only associated with the under-compacted status of unsaturated loess. Therefore, an enough strong seismic loading may cause loess soil suffered dynamic residual deformation incompletely to generate residual strain again.

There is an indistinct relationship between dynamic stress and dynamic residual strain because the residual strain has relations not only with the loading intensity, but also with the structure strength of soil mass. For any field of natural loess, physical parameters of soil are always so uncertain that test data of dynamic residual strain in laboratory is jointly influenced by external seismic loadings and inherent structure strength of soil. The inherent strength is obviously and absolutely different. Consequently, it is impossible to understand reasonable laws of dynamic residual deformation of unsaturated loess by means of simple analysis based on laboratory data of residual strain under some certain dynamic loadings. These difficulties spur the author to find out new ideas onto the topic of loess dynamic deformation.

The magnitude estimation method provided by authors could reasonably compare the diversity of dynamic residual strain of natural loess regardless of the soil collected from any burial depth or any field because its essential formulas are established based on the physical process and mechanical mechanism of plastic deformation of unsaturated loess under seismic loadings. Although the second relation of dynamic void compression is merely analyzed by an empirical way, the fine relevance between stress ratio and compression value of void ratio (both of new concepts introduced here) based on laboratory data still indicate us the further direction to investigate and establish the reasonable/practical constitutive model of natural loess under external loadings.

\section{ACKNOWLEDGEMENTS}

This work is jointly supported by the Basic
Research Foundation of Earthquake Prediction Institute, China Earthquake Administration (No. 2013IESLZ01) and the National Natural Science Foundation of China (No. 51209186, No.41472297). Meanwhile, we would like to express the deep appreciation to Ms. Jiahui Zhu (M.D Student, School of Philosophy and Sociology, Lanzhou University) for her efforts to revise the text in English.

\section{REFERENCES}

1) Chen, C., Shao, S., Luo, A. and Fang, J. (2013): True triaxial tests on structural effects on strength of intact loess, Chinese Journal of Geotechnical Engineering, 35(12), 2322-2327.

2) Chu, F., Shao, S. and Chen, C. (2014): Experimental research on influences of dry density and vertical stress on soil-water characteristic curves of intact unsaturated loess, Chinese Journal of Rock Mechanics and Engineering, 33(2), 413-420.

3) Deng, J., Wang, L. and Zhang, Z. (2007): Microstructure characteristics and seismic subsidence of loess, Chinese Journal of Geotechnical Engineering, 29(4), 542-548.

4) Gao, G. (1980): Microstructure of loess in China, Science in China (Series A), 23(12), 1203-1208.

5) Lei, X. (1987): Pore type and collapsibility of loess in China, Science in China (Series B), 17(12), 1309-1316.

6) Miao, T., Liu, Z. and Ren, J. (1999): Deformation mechanism and constitutive relation of collapsible loess, Chinese Journal of Geotechnical Engineering, 21(4), 783-787.

7) Qian, J. and Yin, Z. (1996): Principle and calculation of geotechnical engineering (2nd edition), ISBN 7-80124-142-8, China Water Power Press, 499 (in Chinese).

8) Rogers, C.D.F., Dijkstra, T.A. and Smalley, I.J. (1994): Hydroconsolidation and subsidence of loess: studies from China, Russia, North America and Europe, Engineering Geology, 37(2), 83-113.

9) Seed, H.B. and Idriss, I.M. (1971): Simplified procedure for evaluating soil liquefaction potential, Journal of Soil Mechanics and Foundation Engineering Division, ASCE, 97(9), 1249-1273.

10) Shao, S., Xu, P., Wang, Q. and Dai, Y. (2014): True triaxial tests on anisotropic strength characteristics of loess, Chinese Journal of Geotechnical Engineering, 36(9), 1614-1623.

11) Sun, J., Tian, W., Xu, S., Liu, K., Wang, L. and Niu F. (2013): Application of probability analysis method to quantitative evaluation of dynamic settlements of natural loess field, Rock and Soil Mechanics, 34(8), 2158-2164, 2173.

12) Sun, J., Wang, L., Qiu, R. and Wang, J. (2012): A mathematical estimation model for seismic subsidence of loess based on physical-mechanical mechanism, Engineering Mechanics, 29(5), 53-60.

13) Terzaghi, K., Peck, R.B. and Mesri, G. (1996): Soil Mechanics in Engineering Practice (3rd edition), ISBN 978-0-471-08658-1, John Wiley \& Sons, 5.

14) Wang, L., Deng, J. and Huang, Y. (2007): Quantitative analysis of microstructure of loess seismic subsidence. Chinese Journal of Rock Mechanics and Engineering, 26(Supp.1), 3025-3031.

15) Wang, L., Shi, Y. and Liu, X. (2003): Loess Dynamics, ISBN 7-5028-2321-2, Earthquake Press, 47-56 (in Chinese).

16) Wang, L. and Zhang, Z. (1993): A method of estimating the quantity of seismic subsidence in loess deposits during earthquakes, Journal of Natural Disaster, 2(3), 85-94. 